\title{
Research Article \\ Acute effects of prone asanas and Pal's pranayama on myalgia, headache, psychological stress and respiratory problems in the COVID-19 patients in the recovery phase
}

\author{
Gopal Krushna Pal ${ }^{\text {* }}$, Nivedita Nanda ${ }^{2}$, Manoharan Renugasundari ${ }^{1}$, Pravati Pal ${ }^{1}$, Uttareshwar Pachegaonkar ${ }^{3}$ \\ *Professor (Senior Scale), Department of Physiology, Program Director, Advance Center for Yoga, ${ }^{1}$ Department of \\ Physiology, ${ }^{2}$ Department of Biochemistry, JIPMER, Puducherry, India \\ ${ }^{3}$ Sri Aurobindo Society, Puducherry, India
}

(Received: November $2020 \quad$ Revised: December $2020 \quad$ Accepted: December 2020)

*Corresponding author: Gopal Krushna Pal. E-mail: drgkpal@gmail.com

\begin{abstract}
Introduction and Aim: It has been observed that recovery from COVID-19 is delayed due to continuation of myalgia, fatigue, headache and some respiratory problems even after the discharge from the hospital. Reports indicate the improvement of sensory, cardiorespiratory and psychological functions following practice of Prone asanas and Pal's pranayama. Therefore, we conducted a pilot study to assess if practice of asanas in prone posture and slow breathing exercise of Pal's pranayama schedule can facilitate recovery from the COVID-19 illness and alleviate post-recovery complications in these patients.
\end{abstract}

Materials and Methods: This is an interventional pilot study conducted in COVID positive patients. A structured module of prone asanas and Pal's pranayama schedule was given to the COVID positive patients in addition to the routine medical treatments and their stress levels were assessed prior to and after the practice. Also, the acute effects of asana-pranayama schedule on the improvement of cardiorespiratory functions and occurrence of other complications in the recovery phase was recorded.

Results: Following practice of asana-pranayama schedule, the patients recovered faster from myalgia, fatigue, headache and respiratory problems and they had a feeling of well-being. Further, the complications in the recovery phase of COVID-19 were prevented and the intensity of stress was reduced with the practice of asana-pranayama schedule.

Conclusion: This pilot study has shed some light on the early recovery and the prevention of complications in the recovery phase of COVID-19.

Keywords: COVID-19; Prone asanas; Pal's pranayama; Recovery from COVID-19; Perceived stress scale

\section{INTRODUCTION}

I nspite of the fact that $80 \%$ of corona virus disease 2019 (COVID-19) patients recuperate without much medical intervention, older people and individuals with comorbid conditions such as diabetes, hypertension, heart and lung diseases and cancer are at higher risk of developing serious illness (1). It has been reported that COVID mortality is mostly due to acute pneumonia and acute respiratory distress syndrome (ARDS) (2). Further, the convalescence of COVID-19 patients is delayed as a result of persistence of myalgia, weakness, headache and stress-related problems. To date, there is no evidence of any effective treatment for COVID-19. Though the current prophylactic measure by antiviral drugs such as chloroquine or hydroxychloroquine and respiratory therapies are being used, it is found meagre (3). There are several ongoing clinical trials of both western and traditional medicines for development of new therapies and vaccines for COVID-19.

Many clinical trials reinforced the role of prone positioning as an effective therapy in the early stage of $\operatorname{ARDS}(4,5)$. Recent reports have suggested improvement in respiratory functions of COVID-19 patients with prone positioning (6-8). Practice of yoga has been reported to improve pulmonary functions in common obstructive pulmonary disease (COPD) patients (9). The asanas and pranayamas are the major components of yoga (10). As prone position facilities respiratory functions, it is far predicted that prone asanas may also promote respiratory functions. Nevertheless, among yoga techniques, the pranayama training (controlled breathings) has been reported to promote respiratory functions (11). Among various kinds of pranayamas, the chandranadi pranayama (left-nostril breathing) and anulom-vilom pranayama (alternate-nostril breathing) had been proven to have the most useful consequences on autonomic cardiorespiratory functions $(12,13)$. Pal's pranayama, a structured slow breathing exercise that comprises of both chandranadi and anulom-vilom pranayamas has been documented to be powerful in preventing ventricular tachycardia by improving vagal tone which is modulated through the process of respiratory sinus arrhythmia (14).

It was proposed that practice of yogasana and slow pranayama could combat and prevent COVID-19 
illness (15). Though most of the COVID-19 patients recover from fever and get discharged from hospital in about 10 days, many of them continue to suffer from myalgia, headache, anosmia, ageusia and some kind of respiratory deficit for a longer duration. Recently, it has been reported that practice of Pal's pranayama could prevent the development of respiratory problems in a COVID-19 patient, who was a known case of bronchial asthma and hypothyroidism. Also, there was decreased psychological stress in this patient (16). Further, it was reported that practice of Pal's Pranayama could facilitate early recovery from anosmia and ageusia in COVID-19 patients (17). Therefore, in the present study we have assessed the effects of practice of a structured module of prone asanas and Pal's pranayama on the recovery from COVID-19 patients.

Worry of death is a major psychological problem of COVID-19 patients, which they experience once they are diagnosed to have the disease. Further, psychological issues like depression and anxiety continue to disturb them immensely (18). Hence, in this study, we have also assessed the practice of asana-pranayama schedule on psychological stress score of COVID-19 patients.

\section{MATERIALS AND METHODS}

\section{Study Design}

This interventional pilot study was conducted after obtaining approval from Research Advisory Committee and Human Ethics Committee of Sri Aurobindo Society, Puducherry, India, an international organization approved by Government of India for integral research in alternative medicines. Sri Aurobindo Society has an MoU with Jawaharlal Institute of Postgraduate Medical Education and Research (JIPMER), Puducherry for health-related research and development.

\section{Patients and Case Details}

Two COVID-19 patients participated in the study. Their written informed consent was obtained for the study. Both the patients after developing fever were found to be COVID-19 positive and were admitted to a Government notified COVID Center in Puducherry.

The first case was a 27-year-old male resident doctor, admitted to COVID-ward with complaints of fever, myalgia and headache. With antipyretics (Tab. Paracetamol, 500mg 1 b.d) and Vitamin B complex tablets, the resident doctor became afebrile in two days and thereafter antipyretics was stopped. However, severity of myalgia, headache and weakness did not subside, and the resident was in a psychological stress due to intense myalgia and headache. Then, the resident contacted a senior professor for the asana-pranayama schedule about which he came to know from one of his colleague admitted to the same COVID ward a week before and was also recovering well from COVID problems by practicing an asana-pranayama. On the advice of the senior professor, he started practicing the asanapranayama schedule as described below, which was given to him through WhatsApp and his stress level was assessed using Perceived Stress Scale (PSS) before and after the practice of asana-pranayama.

The second case was a 28-year-old female doctoral scholar, admitted with complaints of fever and myalgia to the female COVID ward. She was given tab. paracetamol (500mg 1 b.d.) and Vitamin B complex, and she became afebrile in three days. But she developed severe headache and nasal obstruction in the recovery phase. Also, she had anosmia and difficulty in breathing especially while speaking. There was no relief from these problems after using nasal drops and doing vapor inhalation which made her to go into stress. She came to know from another resident doctor who had speedier recovery from COVID by practicing a yoga schedule given by a senior faculty of her department. She contacted the senior faculty, who is known to be an expert in yoga and naturopathy and sought for help to come out of these problems. The faculty assessed her stress using PSS and provided her an asana-pranayama schedule through her WhatsApp with the full instructions of the procedure. She immediately learned and started practicing the asana-pranayama schedule, which is described below. Her stress level was assessed again after 3 days of practice of asana-pranayama.

\section{Asana-Pranayama Schedule}

In addition to the routine medical treatment provided in the hospital, both the patients continued to practice the following asana-pranayama schedule.

Asanas in prone posture: They practiced eight asanas in prone posture as described in yoga textbook (19) and presented with pictures in our earlier publication (16).

Ardha-shalabhasana (half-locust pose): 2 min.

- Lying flat on abdomen, one leg is raised with full extension at knee with other leg in contact with the floor.

- After $1 \mathrm{~min}$, the same movement is repeated in the opposite leg.

\section{Shalabhasana (locust pose): $1 \mathrm{~min}$}

The procedure is same as ardha-shalabhasana, but instead of raising one leg, both the legs are raised at a time.

\section{Bhujangasana (cobra pose): 2 min}

- Lying flat on abdomen, the head, neck, shoulder and thorax are raised slowly with the support of forelimbs. The head is gently tilted backward. 
- After spending 30 seconds in this position, return slowly to flat prone position and relax the lower back muscles for one min.

Sphinx asana: $3 \mathrm{~min}$

- Lying flat on abdomen, the head is raised with shoulder and chest by bringing the upper arms to the vertical position. Keep elbows, forearms and hands on the floor and relax the whole body.

\section{Makarasana (Crocodile pose): 3 min}

- It is same as the sphinx asana, but instead of keeping the forearm on the ground, the head is supported with forearms with chin resting on the palms of both hands.

Jyestikasana: $3 \mathrm{~min}$

- Lying flat on abdomen with forehead resting on the floor, interlock the fingers and place palms on the back of the head and relax the whole body.

Matsya kridasana (flapping fish pose): 5 min.

- Lying on abdomen with the fingers interlocked under the head, the left leg is bent to bring knee close to the chest. The right leg remains straight.

- Relax the whole body $5 \mathrm{~min}$. The posture may be changed to the opposite side.

Advasana (reversed corpse-pose or opposite shavasana): $5 \mathrm{~min}$

- Lying on the abdomen, both the arms stretched above the head with palms and the forehead resting on the floor. Relax the whole body for $5 \mathrm{~min}$, or as much time possible.

\section{Pal's Pranayama schedule}

The patients practiced slow breathing exercise of Pal's pranayama schedule as described earlier (14, $16,17)$.

- Sitting comfortably in nasika mudra, gently close the right-nostril by the thumb and inhale deeply in the left-nostril while counting $1-5$ in mind and then slowly exhale in the same nostril counting 1-10 in mind or as much as possible (but not more than 10 counts).

- Repeat the same process of breathing in leftnostril.

- Then, inhale deeply in the left nostril counting 1-5 in mind and close the left nostril by the index finger and exhale slowly in the right nostril counting 1-10 in mind and then inhale deeply in right nostril counting 15 in mind and close the right nostril by thumb and exhale slowly in the left nostril counting
1-10 in mind, or as much as possible (but not more than 10 counts).

- This completes one pranayama cycle, which has two rounds of the left-nostril (Chandranadi) breathing, followed by one round of alternate nostril (Anulom-Vilom) breathing.

- In this slow pranayama, expand chest during inspiration and relax the body and mind during each expiration.

- Practice about 12 to 15 cycles of this pranayama.

The asana-pranayama schedule was practiced once in the morning between 7.00 AM to 8.00 AM and once in the evening between 5.00 PM to 6.00 PM, each session lasting about 45 minutes. During other time, patients were advised to lie-down on the bed in matsya-kridasana or adavasana, as and when they felt comfortable in those positions.

\section{RESULTS}

\section{Perceived Stress Scale Score}

The stress level of the male resident assessed by Perceived Stress Scale (PSS) score was found to be 26 before practicing asana-pranayama and was reduced to a score of 7 within 48 hours of practicing asana-pranayama. Thus, PSS was considerably reduced following two days practice of asanapranayama practice. The female scholar got relieved from her stress with a PSS score of 6 which was reduced from her score of 23 in three days practicing of asana-pranayama.

\section{Other Symptoms in Recovery Phase}

Within 24 hours of practice, the resident doctor had substantial decrease in myalgia, headache and muscle fatigue and became fully asymptomatic in two days. The female scholar had significant decrease in her headache and nasal obstruction within 12 hours of practice and was fully cured of these problems in 24 hours. But anosmia continued for another two days which was also reverted to normal after 48 hours. The pulse, blood pressure and respiration including $\mathrm{SpO}_{2}$ were normal in both participants throughout their hospital stay and in the recovery period.

\section{DISCUSSION}

The main objectives of treatment of COVID patients are to expedite recovery, relieve trepidation and stress and prevent the development of respiratory illness, especially ARDS and other complications (3). The main purposes of prone positioning are to improve oxygenation and respiratory mechanics, homogenise the pleural pressure-gradient, the alveolar inflation and the ventilation distribution, increase the lung volume and reduce the amount of atelectatic regions, facilitate the drainage of secretions, and reduce ventilator-associated lung injury (5). Thus, practicing asanas in prone position 
and lying-down in advasana (prone-shavasana) for a longer time might have improved the respiratory functions and prevented respiratory problems in these patients. Though there are many prone asanas, we have selected only those asanas that a COVID-19 patient can perform. Further, these asanas help in strengthening the muscles of neck, thoracic cage, abdominal wall and pelvic wall and facilitate the functioning of respiratory apparatus (20). Among them, jyestikasana, matsya-kridasana and advasana are primarily for relaxing the body in prone position and decreasing the stress level of the patient. These asanas also relax the muscles of the body and reduces physical and mental tensions.

Practice of pranayamic breathing has been shown to be effective in improving cardio-pulmonary and autonomic functions (21). In Pal's pranayama schedule, which is a combination of chandranadi (left-nostril) with anulom-vilom (alternate-nostril) breathings, the duration of expiration is more prolonged to further strengthen the cardiopulmonary functions (14). As slow nostril breathing has been reported to promote vagal tone while strengthening the sympathovagal balance (22), the increased duration of expiration in this slow pranayamic breathing might have further potentiated the vagal tone and improved oxygen uptake and oxygenation of tissues of the body. Increased vagal tone is known to improve oxygenation and pulmonary functions in hypoxic human beings (23). Further, in slow breathings, the greater expansion of chest wall and increased afferent discharge from thoracic cage and lungs improves the functions of the breathing apparatus, as documented earlier (20-22). Nevertheless, with the practice of this asanapranayama schedule, the body-mind of the subject gets relaxed, in addition to improvement of cardiorespiratory functions.

Both the patients recovered early and smoothly from COVID-19. It is reported that fear (20\%), nervousness (18\%), sadness (18\%), anxiety disorders and sleep disorders are common in COVID-19 patients (1-3). It was reported that there will be decrease in stress level and increase in cardiovascular functions following practice of slow breathing exercises $(24,25)$. Therefore, as observed in these two cases, the asana-pranayama practice not only facilitated their recovery from COVID-19, but also reduced the psychological distress in them. Also, it prevented the development of complications in the post-recovery period.

As there are no expenses involved in these asanapranayama practices, the intervention being noninvasive and non-pharmacological, and could be performed without difficulty by all age groups, it will be less cost-effective, and there may be wider acceptability. However, controlled trials should be conducted to assess the consistency and reproducibility of these practices.

\section{CONCLUSION}

From the results of this preliminary report, we conclude that practice of asana-pranayama schedule has acute effects in reducing the severity of symptoms and psychological stress and prevents the complications in the recovery phase of COVID-19 patients. Thus, asana-pranayama schedule could be promising in facilitating recovery, reducing psychosomatic stress, and improving the general health of the COVID-19 patients. However, studies should be conducted on a large sample size to assess its additional benefits.

\section{ACKNOWLEDGEMENTS}

Authors acknowledge the sincere co-operation in regular practice of the asana and pranayama programme. Authors also acknowledge the logistic support provided by Shri Vijay ji, the Chief Executive of Sri Aurobindo Society for the conduct of this study.

\section{CONFLICT OF INTEREST}

Authors declare they have no conflict of interest.

\section{REFERENCES}

1. Yang, X., Yu, Y., Xu, J., Shu, H., Xia, J., Liu, H., et al., Clinical course and outcomes of critically ill patients with SARS-CoV-2 pneumonia in Wuhan, China: a singlecentered, retrospective, observational study. Lancet Resp Med. 2020; 8(5): 475-481.

2. Li, L. Q., Huang, T., Wang, Y. Q., Wang, Z. P., Liang, Y., Huang, T. B., et al., 2019 novel coronavirus patients' clinical characteristics, discharge rate and fatality rate of meta-analysis. J Med Virol. 2020; 92(6): 577-583.

3. Pascarella, G., Strumia, A., Piliego, C., Bruno, F., Buono, R. D., Costa, F., et al., COVID-19 diagnosis and management: a comprehensive review (Review). J Intern Med. 2020; 288(2): 192-206.

4. Scholten, E. L., Beitler, J. R., Prisk, G. K., Malhotra, A. Treatment of ARDS with prone positioning. Chest. 2017; 151(1): 215-224.

5. Gattinoni, L., Taccone, P., Carlesso, E., Marini, J. J. Prone position in acute respiratory distress syndrome. Rationale, indications, and limits. Am J Respir Crit Care Med. 2013; 188(11): 1286-1293.

6. Golestanieraghi, M., Mahmoodpoor, A. Early application of prone position for management of Covid-19 patients. J Clin Anesth. 2020; 66: 109917.

7. Elkattawy, S., Noori, M. A case of improved oxygenation in SARS-CoV-2 positive patient on nasal cannula undergoing prone positioning. Resp Med Case Rep. 2020; 30: 101070.

8. Cohen, D., Wasserstrum, Y., Segev, A., Avaky, C., Negru, L., Turpashvili, N., et al., Beneficial effect of awake prone position in hypoxaemic patients with COVID-19: case reports and literature review. Intern Med. 2020; 50(8): 9971000 .

9. Soni, R., Munish, K., Singh, K., Singh, S. Study of the effect of yoga training on diffusion capacity in chronic obstructive pulmonary disease patients: A controlled trial. Int J Yoga. 2012; 5(2): 123-127.

10. Saraswati, S. S. Prone asanas. In: Asana pranayama mudra bandha. 4th Edition, Yoga publication trust, India, 2002.; p. $2-11$. 
11. Pal, G. K., Velkumary, S., Madanmohan. Effect of shortterm practice of breathing exercises on autonomic functions in normal human volunteers. Ind J Med Res. 2004; 120(2): 115-121.

12. Bal, B. S. Effect of anulom vilom and bhastrika pranayama on the vital capacity and maximal ventilatory volume. J Phys Educ Sport Manag. 2010; 1(1): 11-15.

13. Singh, S., Gaurav, V., Parkash, V. Effects of a 6-week nadishodhana pranayama training on cardio-pulmonary parameters. J Phys Educ Sport Manag. 2010; 2: 44-47.

14. Pal, G. K. Disappearance of ventricular ectopics following 15-day practice of Pal's pranayama schedule. Int J Clin Exp Physiol. 2018; 5(2): 105-107.

15. Pal, G. K. Yoga to Combat and Prevent COVID-19. Int J Clin Exp Physiol. 2020; 7(2): 46-7

16. Pal, G. K., Nanda, N., Renugasundari, M., Pal, P. Prone Asanas and Pal's Pranayama could Facilitate Recovery and Prevent Psychosomatic Stress and Complications of COVID-19. Yoga Phys Ther Rehabil 2020; 5: 1078. DOI: 10.29011/2577-0756.001078.

17. Pachegaonkar, U., Rajesh, D. R. Early Recovery from Anosmia and Ageusia of COVID-19 by Practice of Slow Breathing of Pal's Pranayama Schedule: A Brief Communication. Int J Clin Exp Physiol. 2020; 7: 119-121.

18. Balachandar, V., Mahalaxmi, I., Subramaniam, M., Kaavya, J., Kumar, N. S., Laldinmawii G, et al., Follow-up studies in COVID-19 recovered patients - is it mandatory? Sci Total Environ. 2020; 729: 139.

19. Pal, G. K., Pal, P., Nivedita, N. Physiology of yoga and prevention of aging. In: Comprehensive textbook of medical physiology. $2^{\text {nd }}$ Edition, Jaypee Publications; 2019. p. 13591376.

20. Chanavirut, R., Khaidjapho, K., Jarce, P., Pongnaratorn, P. Yoga exercise increases chest wall expansion and lung volumes in young healthy Thais. FASEB J. 2006; 20(5): A1257.

21. Hakked, C. S., Balakrishnan, R., Krishnamurthy, M. N. Yogic breathing practices improve lung functions of competitive young swimmers. J Ayurved Integr Med. 2017; 8(2): 99-104.

22. Pal, G. K., Agarwal, A., Karthik, S., Pal, P., Nanda, N. Slow yogic breathing through right and left nostril influences sympathovagal balance, heart rate variability, and cardiovascular risks in young adults. North Am J Med Sci. 2014; 6(3): 145-151.

23. Ito, S., Sasano, H., Sasano, N., Hayano, J., Fisher, J. A., Katsuya, H. Vagal nerve activity contributes to improve the efficiency of pulmonary gas exchange in hypoxic humans. Exp Physiol. 2006; 91(5): 935-941.

24. Naik, G. S., Gaur, G. S., Pal, G. K. Effect of Modified Slow Breathing Exercise on Perceived Stress and Basal Cardiovascular Parameters. Int J Yoga. 2018; 11(1): 53-58.

25. Pal, G. K., Agarwal, A., Karthik, S., Pal, P., Nanda, N. Slow yogic breathing through right and left nostril influences sympathovagal balance, heart rate variability, and cardiovascular risks in young adults. North Am J Med Sci 2014; 6: 145-51. 